\title{
Abordagem atual aos aneurismas do topo da artéria basilar
}

\author{
Paulo Henrique Pires Aguiar', Adriana Tahara², Patricia Lure Fuke'3, \\ Alexandros Theodoros Panagopoulos ${ }^{4}$, José Carlos Esteves Veiga ${ }^{5}$ \\ Divisão de Neurocirurgia, Hospital São Camilo, São Paulo, Brasil \\ Divisão de Neurocirurgia, Hospital Santa Paula, São Paulo, Brasil \\ Divisão de Neurocirurgia, Faculdade de Ciências Médicas da Santa Casa de São Paulo, Brasil
}

\section{RESUMO}

Objetivo: Revisão crítica das abordagens atuais para aneurismas do topo da artéria basilar. Análise dos aspectos anatômicos e angiográficos relevantes para cirurgia. Métodos: Revisão crítica da literatura e relato da experiência dos autores. Resultados: Os principais acessos para aneurismas de topo de basilar de acordo com sua posição em relação ao dorso da sela são: Kawase transpetroso para os aneurismas baixos, transcavernoso para os médios e temporopolar para os altos. A monitoração eletrofisiológica e com doppler pode minimizar complicações isquêmicas por clipagem prolongada, estenose ou fechamento inadvertido de perfurantes. Neuroproteção e hipotermia devem ser consideradas em lesões gigantes e complexas. Lesões complexas geralmente excedem ao tratamento endovascular. Conclusão: Existe uma tendência na literatura a considerar o tratamento cirúrgico dos aneurismas como método ultrapassado; entretanto a tecnologia de novas formas de proteção cerebral, técnicas microcirúrgicas e evolução dos clipes são indispensáveis para lidar com o cérebro em condições ruins na fase aguda. Os procedimentos endovasculares não drenam hematomas, não tratam hidrocefalia nem retiram coágulos das cisternas para prevenir o vasoespasmo. Entretanto é necessário um seguimento a longo prazo para uma avaliação mais precisa. O neurocirurgião deve dominar as duas opções de tratamento e ser hábil para indicar o tratamento mais apropriado.

\section{PALAVRAS-CHAVE}

Aneurisma intracraniano. Aneurisma da artéria basilar.

\section{ABSTRACT}

Approaches to the basilar artery aneurysm

Objective: To review the main approaches to basilar aneurysms and discuss relevant anatomy and angiographic features to choose the appropriate approach in each case. Methods: Literature review and author's experiences are presented. Results: Current main approaches to basilar aneurysms regarding the level of the lesion from sellae dorsum are: Kawase transpetrosous for lower, transcavernous for middle and temporopolar for higher positioned aneurysms. Doppler, electroencephalography, somatosensory and motor evoked potential might minimize incidence of ischemic complications attributable to prolonged temporary occlusion or inadvertent perforator occlusion. Circulatory arrest and hypotermia may be considered for giant and complex aneurysms. The main principle applied is maximization of bone resection, which facilitates the use of surgical instruments and minimizes brain retraction. Complex basilar aneurysms frequently outdo endovascular treatment. Conclusion: There is a tendency in literature to consider the aneurysm surgery as an outdated method; however, technology of intensive care and anesthesia for brain protection, surgical techniques and clips evolution are indispensable for manipulate angry brain in aneurysms surgery after subarachnoid hemorrhage. Endovascular procedures do not remove clots from cisterns to avoid vasospasm, treat hydrocephalus or fenestrate the lamina terminalis to the same purpose; besides, longer follow up is necessary according to the final result. The neurosurgeon must dominate both treatment options and be able to differentiate exact indications.

\section{KEY WORDS}

Intracranial aneurysm. Basilar artery aneurysm.

\footnotetext{
1 Professor livre-docente da Faculdade de Medicina da Universidade de São Paulo.

2 Doutoranda, Faculdade de Medicina da Universidade de São Paulo.

3 Oftalmologista do Hospital São Camilo, São Paulo.

4 Neurocirurgião do Hospital São Camilo, São Paulo.

5 Professor livre-docente da Faculdade de Ciências Médicas da Santa Casa de São Paulo.
} 


\section{Introdução}

A abordagem cirúrgica para os aneurismas de topo de basilar baseia-se nas características angiográficas, observando-se a localização em relação ao dorso selar, orientação do domus e complexidade morfológica (Figuras 1, 2, 3 e 4). ${ }^{7}$ A avaliação prévia da relação óssea do aneurisma com tomografia de cortes finos também é importante..$^{7,13,31,32}$ Os acessos laterais à circulação posterior exigem conhecimento anatômico intra e extradural da região do forâmen magnum. Pelo menos cinco pontos anatômicos orientam a cirurgia: $:^{7,13,22,23,50,51,66,69}$ (1) a entrada da artéria vertebral na dura-máter; (2) a veia emissária condilar posterior; (3) a borda medial da região distal do seio sigmoide; (4) o canal do hipoglosso; (5) o tubérculo jugular. Esses pontos anatômicos devem ser estudados em cada paciente, de forma a individualizar o acesso.

\section{Variações anatômicas dos aneurismas da artéria basilar}

Os aneurismas da circulação posterior constituem aproximadamente $10 \%$ dos aneurismas intracranianos. ${ }^{21}$

Mac Donald e col. ${ }^{37}$ analisaram seis pacientes com aneurismas na circulação posterior e avaliaram 30 ossos temporais comparando o ganho em exposição da artéria basilar com a retirada óssea nos acessos subtemporal anterior e transpetroso medial. Também foi quantificado esse ganho no terço superior da artéria basilar, no mesencéfalo ventral, na ponte e na região posterior do seio cavernoso. Concluíram que quanto maior o ganho ósseo, mais direto o acesso e menor a morbidade. ${ }^{37} \mathrm{Em}$ nossa análise morfométrica, observamos um ganho de $1 \mathrm{~cm}$ a $1,5 \mathrm{~cm}$ da artéria basilar, clivus e ponte na aplicação dessa técnica em relação à técnica-padrão do acesso subtemporal.

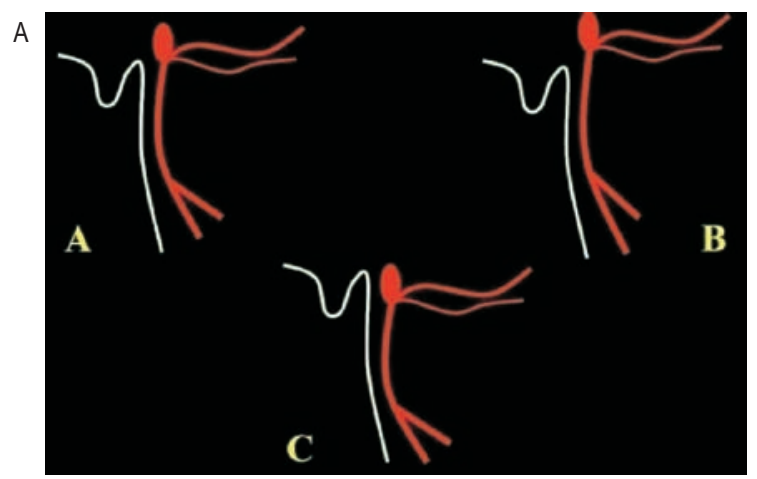

Figura $1 A-$ Esquema em perfil da relação do aneurisma com o dorso da sela túrcica.

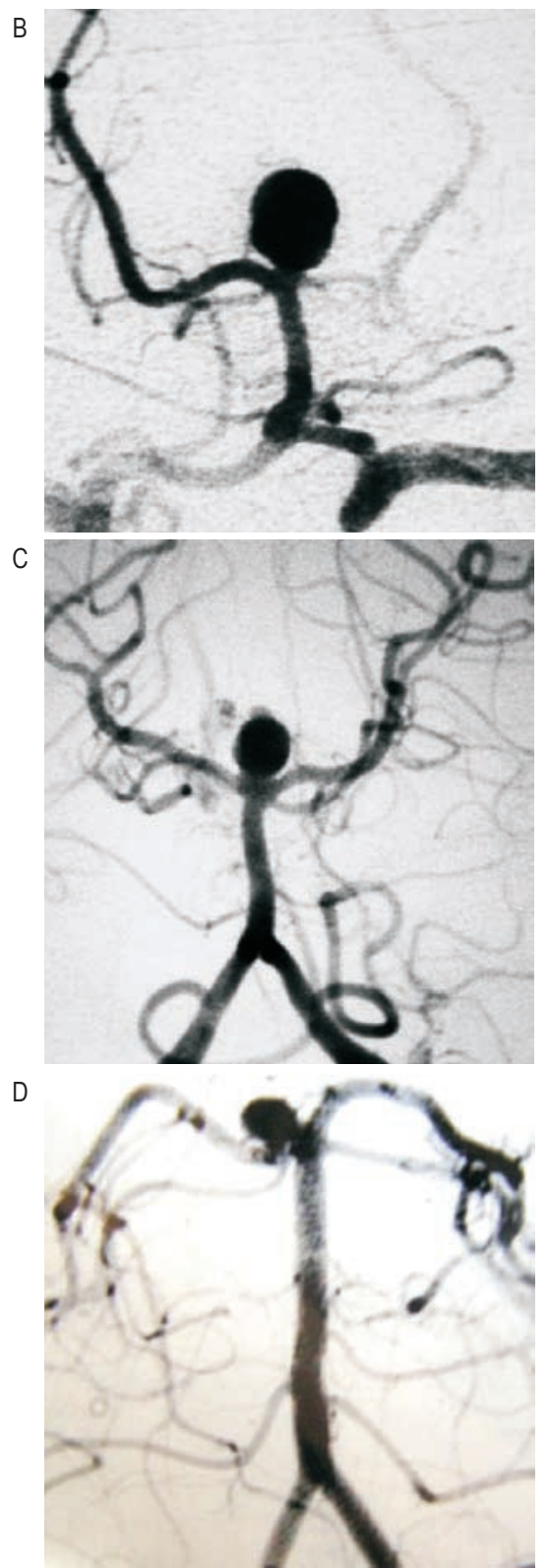

Figuras - 1B: Aneurisma em posição baixa no dorso, 1C: Aneurisma na altura do dorso, 1D: Aneurisma acima do dorso.

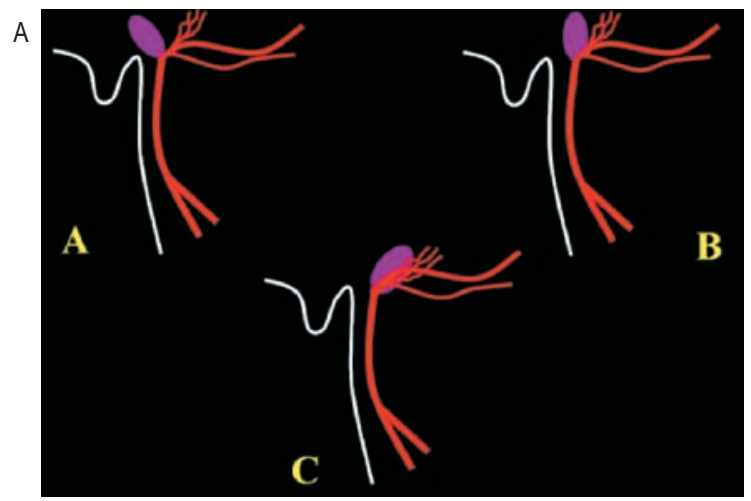

Figura 2A-Representação esquemática da orientação do aneurisma. 
B

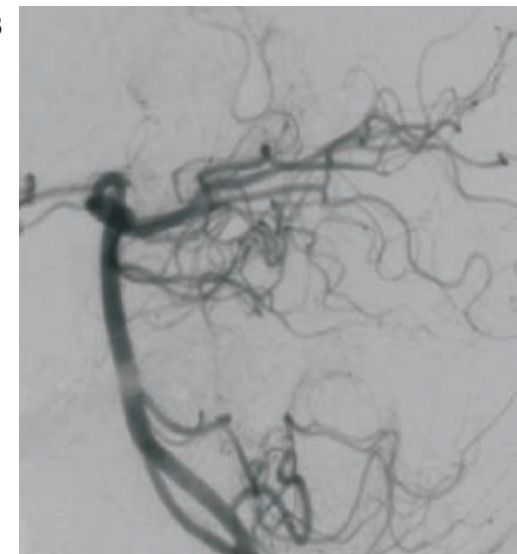

C

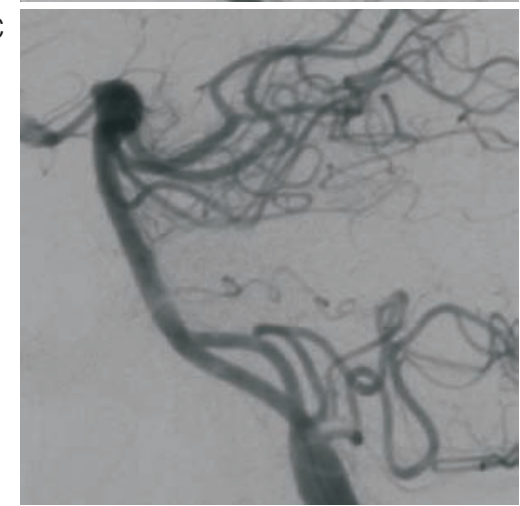

D

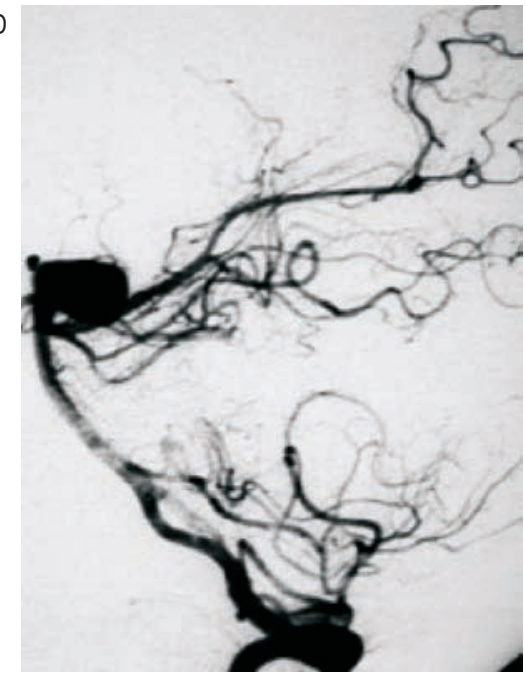

Figuras - 2B: Domo voltado anteriormente, 2C: Domo centrado, 2D: Domo voltado posteriormente.

Pai e col., ${ }^{42}$ em estudo anatômico com 25 encéfalos, verificaram que em $52 \%$ das peças a artéria basilar tinha trajeto curvo e alongado, sendo reta nos demais. O diâmetro da artéria basilar variou de $3 \mathrm{~mm}$ a $7 \mathrm{~mm}$ (média de $4,3 \mathrm{~mm}$ ), com comprimento de $24 \mathrm{~mm}$ a 35 $\mathrm{mm}$ (média de 24,9 $\mathrm{mm}$ ). A origem da artéria basilar situa-se na altura do sulco pontomedular ou $10 \mathrm{~mm}$ abaixo (média de 3,1 mm). A bifurcação está $2 \mathrm{~mm}$ a 11 $\mathrm{mm}$ abaixo dos corpos mamilares (média de 5,6 $\mathrm{mm}$ ).

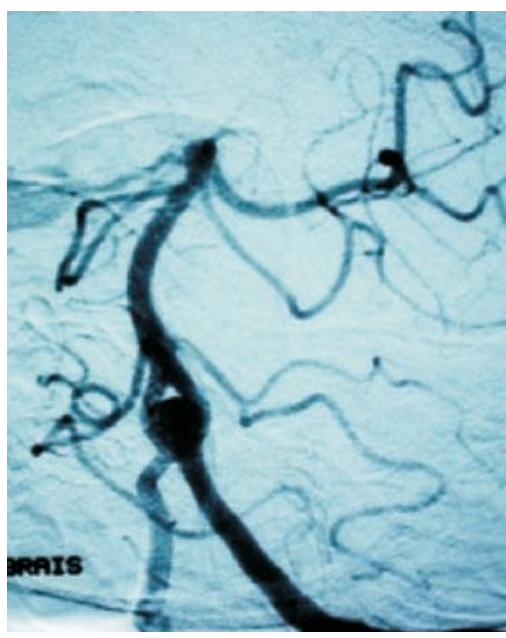

Figura 3 - Angiografia digital do sistema vertebrobasilar com fenestração no terço inferior da artéria basilar. Aneurisma na fenestração e no topo da artéria basilar.

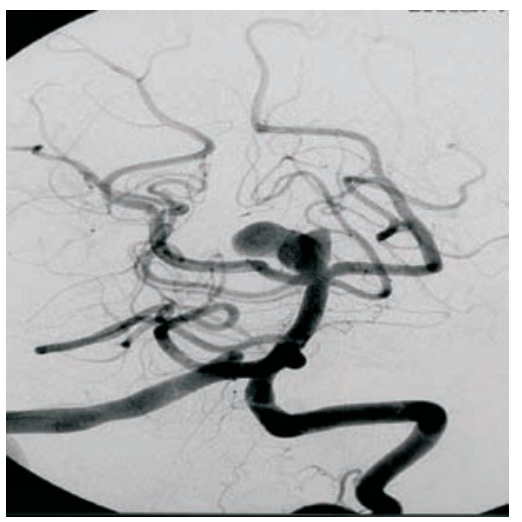

Figura 4-Aneurisma complexo do topo da basilar e na origem da cerebelar anterior inferior à esquerda.

Fenestrações, duplicações ou hipoplasia dessa artéria também foram observadas. A artéria basilar origina perfurantes paramedianas e circunferenciais que suprem a maior parte da ponte e mesencéfalo. Outros ramos maiores incluem a artéria cerebelar anterior inferior (ACAI), a artéria cerebelar superior (ACS) e, algumas vezes, a artéria auditória interna. A quantidade dos ramos paramedianos varia de um a cinco (média de 3,5) à direita, e de um a sete, à esquerda (média de 3,8 ). Os ramos circunferenciais orientam-se lateralmente, para região lateral da ponte e pedúnculos cerebelares. Em quantidade, variam de um a cinco à direita e de um a sete à esquerda. ${ }^{42}$ As artérias talamoperfurantes raramente se originam da bifurcação da artéria basilar, mais comumente originam-se da porção P1 da artéria cerebral posterior.

Torche e col., ${ }^{68} \mathrm{em}$ outro estudo anatômico com 20 encéfalos não fixados, encontraram o comprimento da artéria basilar, entre a artéria cerebelar superior e 
a junção vertebrobasilar, de $28,1 \mathrm{~mm} \pm 1,35 \mathrm{~mm}: 9$ (45\%) com percurso reto, 7 (35\%), curvo, e $4(20 \%)$, tortuoso. ${ }^{68} \mathrm{O}$ número total de perfurantes foi de 340 , média de 17 por cérebro. Cento e dezoito $(34,7 \%)$ medianos e $222(65,2 \%)$ laterais. Ramos medianos apresentaram comprimento médio de $5,8 \mathrm{~mm} \pm$ $1,25 \mathrm{~mm}$, enquanto o dos ramos laterais variaram de $16 \mathrm{~mm} \pm 1,25 \mathrm{a} \pm 1,58 \mathrm{~mm} \cdot{ }^{68}$

O conhecimento da anatomia interpeduncular é fundamental. ${ }^{35,36}$ A membrana de Liliequist, membrana pontomesencefálica medial e lateral formam as paredes da cisterna interpeduncular (Figura 5). A membrana da bifurcação da basilar, perfurada posterior e as trabéculas de aracnoide preenchem a cisterna. ${ }^{35,36}$

O forâmen de Vicq d'Azyr (FVA) é uma área triangular localizada rostralmente na fissura mediana da medula oblonga. Ela é penetrada por pequenos vasos do eixo vertebrobasilar que nutrem núcleos e tratos vitais da ponte. ${ }^{39}$

Mercado e col., ${ }^{39} \mathrm{em}$ estudo com 21 encéfalos fixados, encontraram um total de 286 perfurantes, 13,6 por encéfalo, variando de 7 a 22 . Originavam-se de um total de 73 troncos principais, cerca de 3,5 por encéfalo, variando de 2 a 5 . As perfurantes se originavam principalmente da artéria basilar em 41 (56,1\%); artéria espinal ventral em $12(16,4 \%)$ e artéria vertebral esquerda em 8 (11\%). Os diâmetros das artérias vertebrais esquerda e direita foram respectivamente 3,2 $\mathrm{mm}$ e $2,9 \mathrm{~mm}$, da basilar foi de $4,1 \mathrm{~mm}$, e das artérias espinais ventrais, $6 \mathrm{~mm}$.

Youssef e col. ${ }^{72}$ mensuraram o ganho em exposição antes e após a clinoidectomia anterior, mobilização da artéria carótida e clinoidectomia posterior em cadáve- res. A distância carótida-oculomotor anterior aumentou de $7,1 \mathrm{~mm}$ (variação entre $5 \mathrm{~mm}$ e $10 \mathrm{~mm}$ ) para 10,1 $\mathrm{mm}$ (variação entre $7 \mathrm{~mm}$ e $15 \mathrm{~mm}$ ) após a expansão da abordagem. A distância carótida-oculomotor posterior aumentou de $12,7 \mathrm{~mm}$ (variação entre $9 \mathrm{~mm}$ e $18 \mathrm{~mm}$ ) para $16,1 \mathrm{~mm}$ (variação entre $11 \mathrm{~mm}$ e $22 \mathrm{~mm}$ ). A artéria basilar teve exposição adicional de 4,2 mm (variação entre $1 \mathrm{~mm}$ e $13 \mathrm{~mm}$ ) para $7 \mathrm{~mm}$ (variação entre $3 \mathrm{~mm}$ e $15 \mathrm{~mm}$ ). A clinoidectomia anterior e a mobilização da artéria carótida ampliam o espaço carótida-oculomotor em $44 \%$ anteriormente e $28 \%$ posteriormente. A clinoidectomia posterior aumenta a exposição da artéria basilar em $69 \%$. A exposição mais ampla do campo superficial, janela carótida-oculomotor e basilar facilita a clipagem do aneurisma com controle proximal.

\section{Técnica cirúrgica}

Preferimos o acesso pterional para os aneurismas de topo de basilar. ${ }^{60,71}$ A cabeça é fixada em três pontos numa cabeceira Sugita ${ }^{\circledR}$ ou Mayfield ${ }^{\circledR}$ com um pino no processo mastoide e dos contralaterais, atrás da linha do cabelo na linha temporal superior. A cabeça é rodada $30^{\circ}$ e defletida de $15^{\circ}$ a $20^{\circ}$. A incisão se estende do tragus à linha média, atrás da linha do cabelo e de forma curvilínea anteriormente, preservando o ramo frontal da artéria temporal superficial e ramo frontal do nervo facial. O músculo temporal é incisado na linha temporal e afastado inferiormente com ganchos. O músculo é incisado posteriormente, paralelo à incisão para prevenir
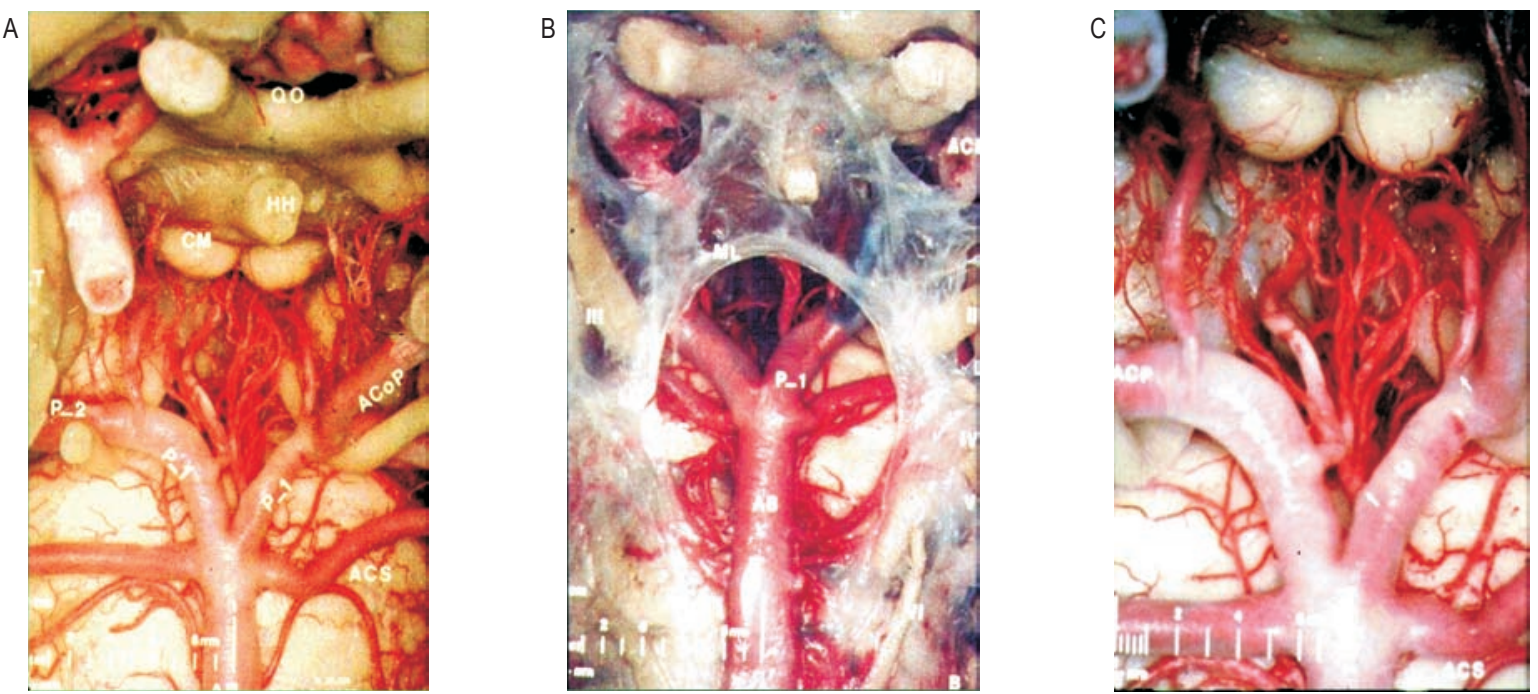

Figura 5-A-C: QO quiasma óptico, HH haste da hipófise, CM corpos mamilares, LT lobo temporal, ACoP (tipo fetal) artéria comunicante posterior, AB artéria basilar, ML membrana de Lilliequist, ACI artéria carótida interna, ACP artéria cerebral posterior, ACS artéria cerebelar superior, Artérias talamoperfurantes (seta). Fusão caudal e simétrica da artéria basilar 
o trismo. A craniotomia é realizada com três pontos: key hole, escama frontal e na sutura escamosa, expondo a fissura silviana e o giro temporal superior. A asa do esfenoide é retirada até a parte lateral da fissura orbital superior, seguida de clinoidectomia extradural anterior. A dura-máter é aberta com uma incisão curvilíena e refletida anteriormente (Figuras 6, 7, 8 e 9).

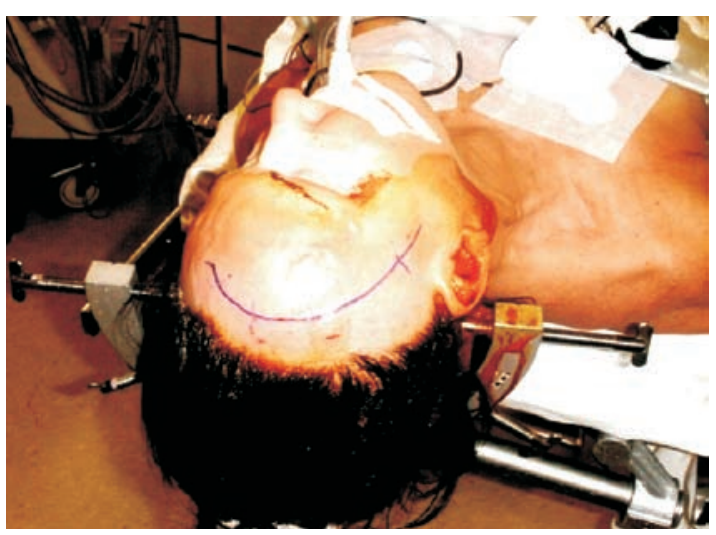

Figura 6 - Posição para pterional direita, fixação na cabeceira de Sugita ${ }^{\circledR}$

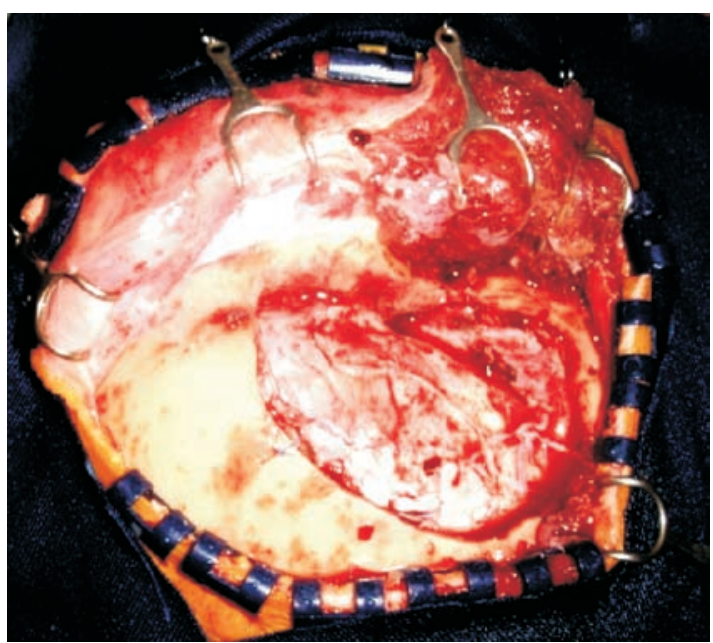

Figura 7 -Craniotomia pterional.

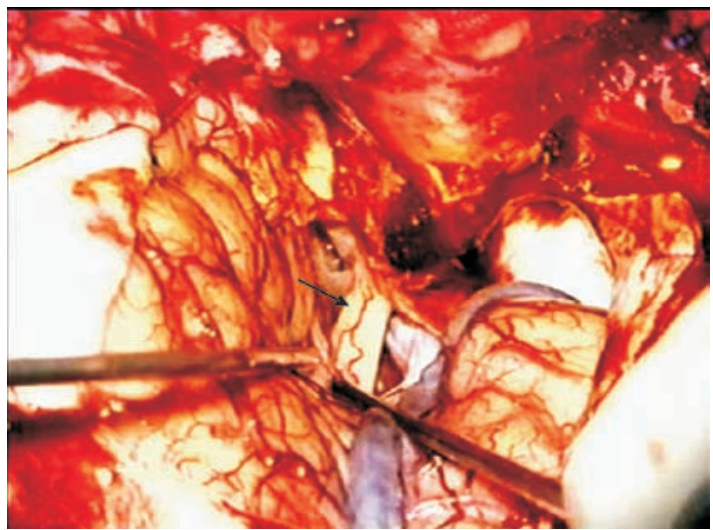

Figura 8 - Visualização do nervo óptico intradural (seta) após orbitotomia extradural.

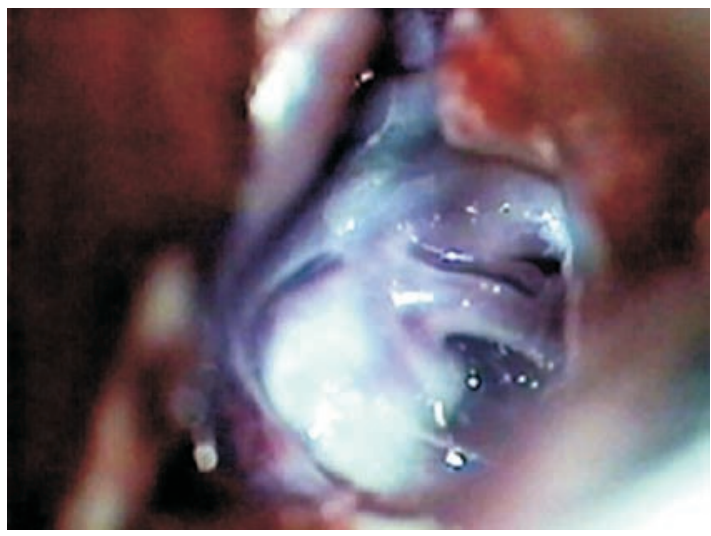

Figura 9 - Aneurisma do topo da basilar.

\section{Discussão}

Em 1961, Charles Drake ${ }^{14}$ descreveu o tratamento dos aneurismas de topo de basilar pelo acesso subtemporal em quatro pacientes.

Suzuki ${ }^{61-64}$ descreveu o acesso bifrontal mediano para cisterna interpeduncular entre os nervos olfatórios e ópticos, com bons resultados também para aneurismas gigantes da basilar. Atualmente, o acesso é utilizado para tratar tumores selares grandes e meningiomas do tubérculo da sela, sendo abandonado para os aneurismas.

Rice e col. ${ }^{49}$ reportaram uma casuística de 167 pacientes operados de 179 aneurismas vertebrobasilares não rotos com mais de $25 \mathrm{~mm}$ de diâmetro. Cento e sessenta foram tratados por clipagem direta, e 19, por métodos alternativos. Cinquenta e três (32\%) tinham um aneurisma e 114 (68\%) tinham múltiplos aneurismas ou malformação arteriovenosa associada, tratada concomitantemente. Muitas das lesões associadas eram rotas. Tiveram 78 complicações pós-operatórias: 23 sistêmicas, 7 hematomas, 6 injúrias por retração cerebral, 5 meningites assépticas, 3 episódios de crises, 3 infecções de feridas. Múltiplas complicações em 23. Setenta e um pacientes se recuperaram completamente sem sequelas. Houve seis resultados ruins e um óbito, taxa de morbimortalidade de $4,2 \%$ na casuística total e $2,4 \%$ para os aneurismas da circulação posterior.

Steinberg e col..$^{59}$ apresentaram uma série de 201 pacientes com aneurismas do sistema vertebrobasilar em que o colo não poderia ser clipado diretamente, submetendo-os à oclusão da artéria basilar ou vertebral. Oitenta e três aneurismas do topo da basilar, 46 do tronco da basilar, 35 da junção vertebrobasilar e 37 da vertebral; $87 \%$ destes eram lesões gigantes $(>2,5 \mathrm{~cm})$. Em 85 foram realizadas oclusões basilares altas, em 41, oclusões basilares baixas, em 29 , oclusões vertebrais bilaterais, e em 48 , unilaterais. O seguimento clínico variou de 1 a 23 anos, média de 9,5 anos. Resultados 
a longo prazo foram excelentes em $68 \%$, bom em $5 \%$, pobre em $3 \%$ e de óbito em $24 \%$. Os resultados, de acordo com a localização, foram: excelente ou bom em $64 \%$ dos aneurismas do topo basilar, $76 \%$ do tronco basilar, $74 \%$ da junção vertebrobasilar e $87 \%$ nos aneurismas da artéria vertebral. $\mathrm{O}$ resultado também variou dependendo do estado pré-operatório: $86 \%$ dos pacientes clinicamente em boas condições obtiveram excelente resultado. O tamanho das artérias comunicantes posteriores foi um bom indicador da tolerância à oclusão basilar $(\mathrm{p}<0,05)$. Trombose do aneurisma foi conseguida em $78 \%$ dos pacientes. Vinte e seis pacientes $(13 \%)$ pioraram por causa de isquemia vertebrobasilar na primeira semana pós-operatória; trombose e embolismo foram mais implicados que insuficiência hemodinâmica. No primeiro mês, houve hemorragia subaracnoide em 14 pacientes, vasoespasmo em 5, trauma cirúrgico em 7 , muitos com boa recuperação. Complicações isquêmicas tardias ou piora neurológica por efeito de massa persistente ocorreu em $4 \%$ dos pacientes com trombose do aneurisma 6 semanas a 18 meses após a oclusão. Dentre 43 pacientes com aneurismas incompletamente trombosados, $67 \%$ evoluíram com deterioração neurológica por hemorragia subaracnoide, compressão progressiva do tronco, isquemia do tronco, fatais em $86 \%$ das complicações.

Peerless e col. ${ }^{44}$ apresentaram uma série de 1.767 pacientes com aneurismas da circulação vertebrobasilar, a maioria operada 14 dias ou mais após a hemorragia subaracnoide. Dos pacientes com boa gradação pré-operatória (Botterell 1 ou 2), bom resultado foi obtido em $80 \%$. Setenta por cento dos pacientes em grau 4 ficaram significantemente sequelados, e todos, exceto um, pacientes em grau 5 morreram. Treze por cento dos pacientes desenvolveram vasoespasmo clínico. Esses autores recomendam cirurgia precoce para pacientes em bom estado clínico e com lesões não complexas e para pacientes com hemorragias recorrentes.

Drake e Peerless ${ }^{15}$ observaram a pobreza de informações sobre aneurismas fusiformes. De 120 pacientes tratados cirurgicamente, em 25 estes localizavam-se na circulação anterior, e 95, na circulação posterior. Seis pacientes tinham aterosclerose, e três, outra arteriopatia. Efeito de massa ocorreu em $50 \%$ e hemorragia em $20 \%$. Oito aneurismas causaram ataque isquêmico transitório. Os tratamentos mais aplicados foram oclusão hunteriana proximal e trapping. Em contraste com os aneurismas saculares, os fusiformes necessitam de duas colaterais: uma para circulação distal do vaso ocluído e uma para os vasos perfurantes que se originam do aneurisma. Noventa por cento dos pacientes com esses aneurismas da circulação posterior obtêm bons resultados, $67 \%$ dos aneurismas de vertebral e basilar têm bons resultados, embora $17 \%$ apresentem condições pré-operatórias comprometidas por compressão do tronco.

A face ventral do tronco é cercada anteriormente pelo clivus, posteriormente pelo tronco e lateralmente pela pirâmide petrosa e por nervos cranianos do III ao XII. ${ }^{31,32}$ As patologias neurocirúrgicas extra-axiais dessa área são os aneurismas do sistema vertebrobasilar, tumores benignos como meningiomas clivais, cordomas, condromas, neurinomas trigeminais e tumores epidermoides pré-pontinos. ${ }^{31,32}$

Kawase $^{31}$ descreveu o acesso subtemporal extradural para a margem petrosa. A ressecção de osso da pirâmide petrosa anterior gerou acesso direto à porção baixa da artéria basilar, com mínima retração do lobo temporal e veias pontes. Os autores trataram dois pacientes com aneurismas baixos do tronco da basilar clipando com sucesso os aneurismas. A audição foi preservada em um caso.

Hakuba $^{22}$ minimizou a retração cerebral com exposição otimizada da região selar e interpeduncular por intermédio do acesso orbitozigomático infratemporal. Nove pacientes com aneurismas do topo da basilar e um no segmento $\mathrm{P} 1$ foram abordados por esse acesso com resultados excelentes..$^{22,23} \mathrm{O}$ princípio mais importante que deve ser aplicado é a máxima remoção óssea que proporciona um corredor amplo de acesso com mínima retração cerebral. ${ }^{21}$

Os aneurismas complexos geralmente excedem ao tratamento cirúrgico. ${ }^{34}$

Figueiredo e col., ${ }^{16}$ estudando modelos anatômicos com espécimes injetados, concluíram que o acesso transcavernoso completo aumenta a área de trabalho e a exposição linear da artéria basilar comparado com técnicas mais conservadoras. Após a craniotomia orbitozigomática, o cirurgião trabalha em sentido reverso perfazendo o acesso transcavernoso em cinco etapas: clinoidectomia posterior, abertura do seio cavernoso, clinoidectomia anterior, abertura do anel dural distal e abertura do anel dural proximal.

Krisht e col. ${ }^{34}$ usaram o acesso transcavernoso para aneurismas complexos e gigantes do segmento basilar, pela via pré-temporal transcavernosa, abrindo o trígono oculomotor e removendo os processos clinoide anterior e posterior quando necessário. Um clipe temporário é posicionado no tronco da basilar numa zona livre de perfurantes, proximal à artéria cerebelar superior. ${ }^{34} \mathrm{De}$ acordo com esses autores, paresia transitória parcial ou completa do nervo oculomotor ocorreu em todos os casos (aneurismas grandes ou gigantes em 27 pacientes, dismórficos em 18, bifurcação baixa em 21 pacientes, com domo posteriormente projetado em 11 , dolicoectasia em 3) com total recuperação em todos exceto em um. Na alta hospitalar, o Glascow Outcome Scale (GOS) foi pontuado em 4 ou 5 em $88 \%$ dos pacientes. 
Aos seis meses o Rankin Outcome Scale era 0 a 2 em $92 \%$ dos pacientes.

O acesso subtemporal é uma via importante para os aneurismas de topo de basilar altos. A elevação do tentório aumenta a visibilidade do colo do aneurisma. ${ }^{14,15,25,46,60}$

$\mathrm{O}$ acesso temporopolar para aneurismas da artéria basilar na região da bifurcação foi descrito, em 1980, por $\mathrm{Sano}^{52,53}$ e consiste de uma craniotomia pterional estendida com acesso subtemporal devido à remoção da base do osso temporal. Todo lobo temporal é exposto e afastado para cima e lateralmente para visualização da fossa interpeduncular e cisterna ambiens. Desde então, várias modificações foram propostas. ${ }^{51,52,57} \mathrm{Se}$ o aneurisma do topo de basilar for muito alto, às vezes a remoção temporária do arco zigomático se faz necessária. ${ }^{51,52,57}$

Sato e col.,${ }^{54}$ na tentativa de aumentar o acesso pterional, numa análise anatômica em 10 peças (20 lados) removeram o processo clinoide anterior e o teto do nervo óptico. A artéria carótida interna foi mobilizada medialmente em $6,1 \mathrm{~mm}$ e $0,8 \mathrm{~mm}$. A extensão da prega dural na área do defeito ósseo no teto do canal óptico foi 2,1 mm e a área, 13,6 mm. 2,54 Em dez casos clínicos, o procedimento permitiu espaço para acesso aos aneurismas de topo de basilar sem alteração do fluxo da carótida interna e com resultados satisfatórios. ${ }^{54}$

Paladino e col. ${ }^{43}$ demonstraram que a maioria dos aneurismas supratentoriais e de topo de basilar eram acessíveis pela craniotomia keyhole supraciliar, e que os aneurismas da artéria vertebral e basilar baixos, pela via retrossigmoide. Com resultados similares às técnicas-padrões, obtiveram GOS 5 e 4 em 398 (82,57\%) pacientes com craniotomia keyhole e $116(79,45 \%)$ com técnica-padrão. A taxa de mortalidade no grupo keyhole foi $0,83 \%$ (4 pacientes) e $2,05 \%$ ( 3 pacientes) no grupo standard. ${ }^{43}$ Nós evitamos a craniotomia keyhole para aneurismas complexos e da circulação posterior, porque não há controle completo para eventual ruptura ou para lidar com um cérebro em condições ruins na fase aguda da hemorragia subaracnoidea, embora seja selecionado de acordo com a experiência e familiaridade de cada grupo cirúrgico. ${ }^{43}$

O doppler intraoperatório é um parâmetro importante para cirurgia de aneurismas, pois a preservação da luz vascular é um dos fatores mais importantes que determina o resultado cirúrgico. O doppler é mais prático, mais rápido e menos invasivo que a angiografia intraoperatória. ${ }^{2}$

Potencial evocado somatossensorial e eletroencefalograma são técnicas-padrão para monitoramento do nível de neuroproteção em anestesia e isquemia na oclusão temporária. ${ }^{47} \mathrm{O}$ potencial evocado motor monitora de forma mais sensível a isquemia por oclusão da artéria basilar e perfurantes, minimizando esse tipo de complicação. ${ }^{47}$

Nós preferimos os clipes de Sugita (Figura 10) por sua configuração, força e compatibilidade com a ressonância, embora existam vários outros. ${ }^{18,60}$ Os clipes fenestrados são importantes para aneurismas do sistema vertebrobasilar para preservação da luz dos vasos principais. ${ }^{60} \mathrm{Em}$ 1999, Segawa e col. ${ }^{55}$ apresentaram um clipe interessante com abertura de dentro para fora, de forma que as lâminas do clipe eram vistas no colo do aneurisma; além disso, os clipes podiam ser angulados em qualquer direção no aplicador, fazendo seu posicionamento mais fácil. O aplicador também funcionava como removedor quando o ajuste do clipe se fazia necessário. Em 30 aneurismas operados com esses clipes, não houve complicações devidas a este. Infelizmente, essa tecnologia ainda não é disseminada em outros países.

Sano ${ }^{53}$, em 1966, propôs o acesso transoral para os aneurismas de basilares. Entretanto, a complexidade do acesso e as complicações como fístula de liquor e infecção tornaram-no pouco popular. ${ }^{24,50,51}$
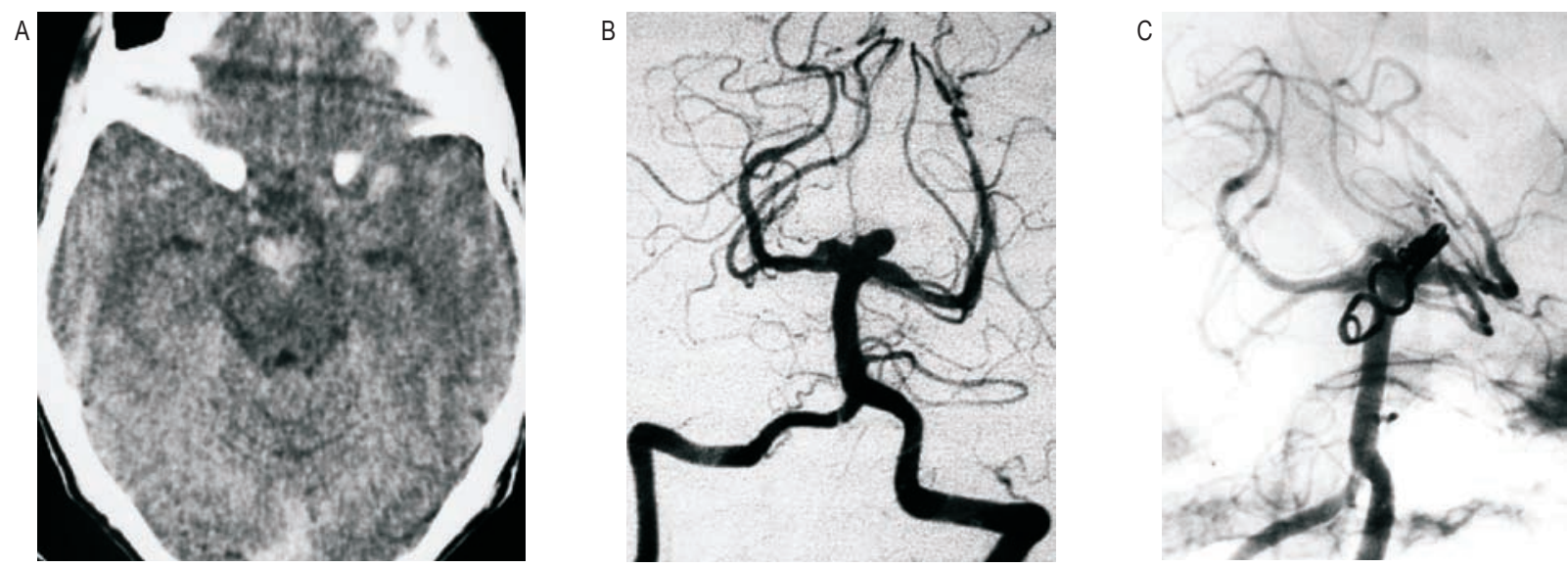

Figura 10 - A: TC de crânio com hematoma interpeduncular, B: Aneurisma do topo da basilar com colo largo, $C$ : Exclusão cirúrgica com perfurantes e vaso principal patentes. 
A restrição do acesso aos aneurismas de topo de basilar e vertebrobasilares da linha média levou ao desenvolvimento de abordagem complexa de base do crânio. ${ }^{22,23} \mathrm{O}$ acesso transcondilar é útil nos aneurismas basilares baixos. Outro acesso proposto foi o retroauricular transpetroso para os aneurismas basilares médios e altos. ${ }^{70} \mathrm{~A}$ via transpetrosa anterior com secção do tentório permite acesso às porções altas do clivus. ${ }^{22}$

Os aneurismas da artéria cerebelar posteorior inferior (ACIP) e basilar acima da junção vertebrobasilar podem ser tratados por um acesso extremolateral.4,11 A ACIP tem um dos trajetos mais tortuosos de forma que um estudo personalizado é necessário. ${ }^{4} \mathrm{O}$ fator mais importante para o acesso é a lateralidade do aneurisma e sua distância do forâmen magno na angiografia digital. Há muitos acessos cirúrgicos para aneurismas baixos do sistema vertebrobasilar. ${ }^{19,20,26,45}$

Tedeschi ${ }^{66}$ defendeu o acesso extremolateral, incluindo a dissecção muscular da porção posterolateral da junção craniocervical com exposição do processo transverso do atlas e triângulo suboccipital, identificação da artéria vertebral entre os processos transversos do atlas e axis, craniectomia ou craniotomia suboccipital com remoção de metade do arco posterior do atlas. ${ }^{13,48,66}$ Para alguns, o arco posterior do atlas não precisa ser removido. ${ }^{48}$

Três modificações em relação ao côndilo occipital são possíveis: acesso transcondilar, supracondilar com extensão paracondilar através da área lateral ao côndilo com acesso à região medial do canal do hipoglosso e tubérculo jugular ${ }^{66}$ e transcondilar com extensão lateral e acesso à parte baixa do clivus e da área pré-medular.

Para os aneurismas baixos da artéria basilar, nós preferimos a posição semissentada, pois evita manipulação do nervo vago, as tonsilas cerebelares são elevadas e o aneurisma é acessado atrás do nervo. ${ }^{40}$

Massad e col. ${ }^{38}$ descreveram a técnica de hipotermia por desvio circulatório por canulação femoral sem abertura do tórax para aneurismas intracranianos complexos. Neuroproteção também pode ser incrementada com uso de barbituratos, além de outras técnicas de hipotermia e desvio circulatório. ${ }^{30,58}$

Os resultados das cirurgias dos aneurismas baixos da artéria basilar são controversos na literatura.

Inamasu e col. ${ }^{27}$ mostraram o resultado a longo prazo de 17 pacientes com aneurismas grandes e gigantes da fossa posterior: 8 casos de aneurismas da bifurcação da basilar, 3 casos do tronco da basilar, 6 casos da artéria vertebral. Oito pacientes foram tratados por via endovascular ou cirurgia. Os outros 9 foram seguidos clinicamente. O resultado foi bom em 6 , moderado em 1 e houve 1 caso de estado vegetativo. Dos seguidos clinicamente, 4 tiveram ruptura fatal e outros dois sofreram agravamento dos sintomas preexistentes rela- cionados aos aneurismas. Só 3 permaneceram intactos. Comparando parâmetros radiológicos dos aneurismas rotos e não rotos, observou-se que as lesões com ruptura subsequente tinham altas taxas de trombos e tendência a aumentar de tamanho. ${ }^{27}$

Clipagem intencional do corpo ou domo de aneurismas de colo largo são eficientes em prevenir ressangramento, embora a oclusão de vasos perfurantes permaneça problemática. A opção da região de clipagem deve ser feita precocemente durante a microcirurgia para preservação dessas perfurantes. ${ }^{65}$

Aneurismas de novo do topo da artéria basilar foram reportados tardiamente por Shimokawa e col..$^{57}$, num período que variou de 7 a 20 anos, média de 12 anos.

Fox e Drake ${ }^{17}$ enfatizaram o uso de embolização com balão destacável para aneurismas basilares complexos como terapia adjuvante.

Os aneurismas intracranianos gigantes têm prognóstico ruim se não tratados..$^{28,41}$ A maioria dos aneurismas pode ser tratada de forma eficiente por embolização endovascular ou clipagem microcirúrgica, mas na fase aguda da hemorragia subaracnoide e com múltiplos aneurismas, a abordagem sequencial multimodal tem melhores resultados. ${ }^{29}$ Aneurismas da artéria basilar associados com fenestração na confluência das vertebrais (Figura 3) ou de morfologia complexa (Figura 4) são de difícil abordagem cirúrgica, e a embolização com coils (Figura 11) é uma boa opção. ${ }^{29}$ Muitos autores consideram o tratamento endovascular a principal opção nos aneurismas da circulação posterior, independentemente da graduação do paciente na escala de Glasgow, de complexidade ou localização. Entretanto, ruptura e outras complicações graves também estão presentes. ${ }^{8}$

Pode-se posicionar um Stent Neuroform ${ }^{\circledR}$ horizontal através do colo largo do aneurisma pela artéria comunicante posterior, em vez de dois stents numa configuração em Y pela artéria basilar. ${ }^{10}$

As taxas de morbimortalidade das embolizações com stents têm caído nos últimos cinco anos. Técnicas de remodeling, refinamento dos coils, balões, cateteres e guias hidrofílicos com pontas de configuração complexa contribuíram para melhores resultados nos procedimentos endovasculares. Vinte e um por cento dos aneurismas foram considerados incompletamente ocluídos imediatamente após a embolização endovascular. ${ }^{12}$ Destes, $1 / 3$ ocluiu-se completamente nos angiogramas de controle após seis meses; $1 / 3$ teve oclusão de mais de $95 \%$ com um pequeno colo residual que permaneceu constante na angiografia de controle após seis meses, um, dois e quatro anos; nenhum ressangrou. ${ }^{12}$

A técnica de remodeling aumentou as possibilidades de tratamento endovascular e ofereceu uma alternativa aos aneurismas com falha no tratamento ou inacessíveis à cirurgia. Estudos a longo prazo ainda são necessários. 
A

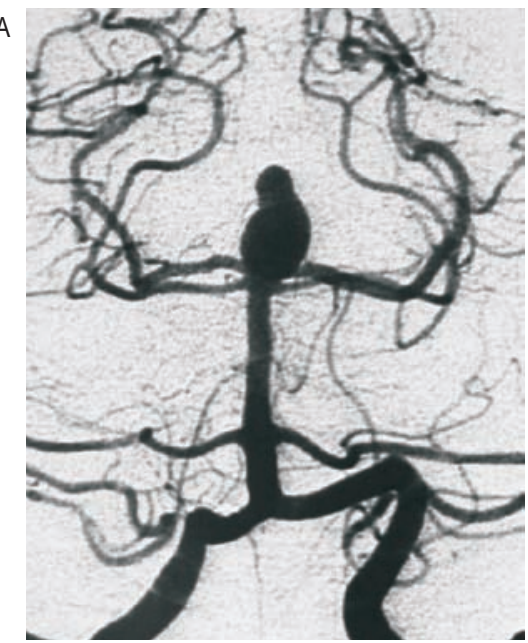

B

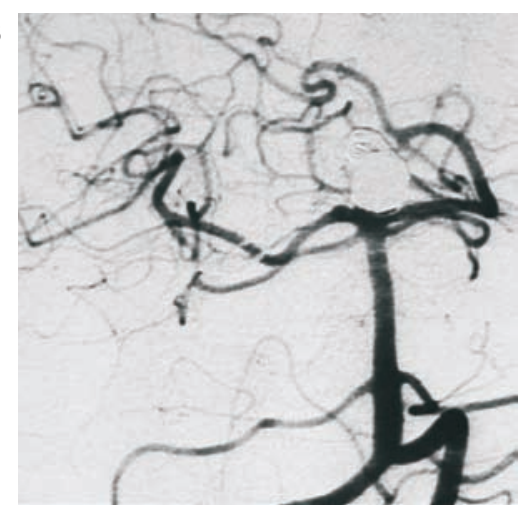

Figura 11 - A: Aneurisma bilobulado do topo da basilar, $B$ : Exclusão da circulação via endovascular com molas destacáveis.

Aletich e col. ${ }^{1}$ reportaram a oclusão com coils em 66 (88\%) de 75 aneurismas; em 8 (11\%), a falha do tratamento ocorreu devida à tortuosidade do vaso ou devida à inadequação dos balões usados. Cinquenta (78\%) de 64 aneurismas tiveram oclusão maior que 95\%, 8 $(12 \%)$ foram parcialmente ocluídos $(<95 \%)$. No total de aneurismas embolizados, 8 aneurismas ocluíram-se completamente e 5 exibiram trombose progressiva. ${ }^{1}$ Em 3 houve crescimento do colo residual. Tratamento cirúrgico foi realizado em 6 aneurismas com falha no tratamento endovascular. Houve 4 pacientes com déficits e 3 mortes relacionadas diretamente ao procedimento. ${ }^{1}$

De 1979 a 1999, Thornton e col. ${ }^{67}$ relataram seis séries cirúrgicas de aneurismas que foram submetidas à angiografia pós-operatória imediata, num total de 1.397 pacientes. Eles estabeleceram dois grupos: 1.370 foram submetidos à angiografia pós-operatória imediata, demonstrando 1.569 aneurismas clipados. Enchimento residual foi observado em 82 aneurismas (5,2\%). Só 124 pacientes com 169 aneurismas tiveram um controle angiográfico a longo prazo. No segundo grupo, 226 pacientes tiveram sintomas recorrentes de hemorragia e efeito de massa mais de 24 anos após a clipagem dos aneurismas ou tiveram achados importantes na angiografia intra e pós-operatória. Eles concluíram que o lapso de informação em relação à frequência de colo residual ou o recrescimento e o controle a longo prazo dos aneurismas tornam difíceis comparações entre tratamento cirúrgico e novos métodos de tratamento.

Em aneurismas grandes e gigantes de colo largo e conformação complexa, onde o tratamento endovascular e cirúrgico têm resultados pobres, o by-pass entre a artéria carótida externa e a circulação posterior pode ser uma solução.

Sekhar e col. ${ }^{56}$ propuseram um protocolo de classificação e tratamento dos aneurismas saculares da circulação posterior: (1) aneurisma menor que $2 \mathrm{~cm}$ com relação domo/colo menor ou igual a 1,5 ; (2) aneurisma maior que $2 \mathrm{~cm}$ ou com relação domo/colo maior que 1,5. No primeiro caso, se o paciente tiver mais que 50 anos de idade, opta-se por tratamento endovascular; se tiver menos que 40 anos, o tratamento de escolha é a cirurgia. Nos pacientes entre 40 e 50 anos, tratamento é individual. Pacientes do segundo grupo com idade menor que 40 anos são operados; se, no entanto, houver algum ramo originando-se do colo, um by-pass pode ser necessário. Pacientes com mais de 50 anos, com aneurismas não rotos, são encaminhados para tratamento endovascular com embolização e balão ou stent como suportes. Nos casos entre 40 e 50 anos, o tratamento é individualizado.

Nos aneurismas fusiformes, se o território do vaso principal tiver uma circulação colateral adequada, deve-se considerar a oclusão do vaso. Se as colaterais forem pobres, opta-se pelo by-pass ou pela reconstrução vascular com posterior exclusão do aneurisma da circulação. As opções de revascularização da ACIP são sutura direta, interposição de enxertos, by-pass com a artéria occipital ou ACIP contralateral. ${ }^{56}$

Apesar da diferença de calibre entre a ACIP e a artéria vertebral, a anastomose entre as duas artérias proporciona uma alternativa. ${ }^{6}$ Resultados favoráveis são obtidos em 90\% dos pacientes com aneurismas da circulação posterior, 60\% nos aneurismas ACIPvertebral e $39 \%$ nos da junção vertebrobasilar-basilar com as técnicas cirúrgica e endovascular. ${ }^{9}$

Existe uma tendência na literatura em considerar o tratamento cirúrgico dos aneurismas intracranianos um método ultrapassado ou mesmo em extinção. ${ }^{3}$ Entretanto, a tecnologia de novos métodos de proteção cerebral, técnicas cirúrgicas e evolução de clipes são indispensáveis para o manejo do encéfalo em condições ruins na fase aguda da hemorragia subaracnoide. Os procedimentos endovasculares não tratam hidrocefalia, não fenestram a lâmina terminalis, não drenam hematomas nem retiram coágulos das cisternas para diminuir a chance de vasoespasmo. Não existem estudos que garantam a oclusão dos aneurismas a longo prazo. ${ }^{73}$ 
O neurocirurgião tem de ser capaz de dominar as duas opções de tratamento e diferenciar as indicações exatas para cada tipo de procedimento.

\section{Referências}

1. Aletich VA, Debrun GM, Misra M, Charbel F, Ausman JI. The remodeling technique of balloon-assisted Guglielmi detachable coil placement in wide-necked aneurysms: experience at the University of Illinois at Chicago. J Neurosurg. 2000;93:388-96.

2. Amin-Hanjani S, Meglio G, Gatto R, Bauer A, Charbel FT. The utility of intraoperative blood flow measurement during aneurysm surgery using an ultrasonic perivascular flow probe. Neurosurgery. 2006;58:305-12.

3. Aussman JI. The death of cerebral aneurysm surgery. Comment. Surg Neurol. 2003;59:338

4. Avci E, Acevedo C, Fossett D. Extreme lateral approach. In: Fossett DT, Caputy AJ, editors. Operative neurosurgical anatomy. New York: Thieme; 2002. p.90-9.

5. Babu RP, Sekhar LN, Wright DC. Extreme lateral transcondylar approach: technical improvements and lesions learned. J Neurosurg. 1994;81:49-59.

6. Benes L, Kappus C, Sure U, Bertalanffy H. Treatment of a partially thrombosed aneurysm of the vertebral artery by aneurysm trapping and direct vertebral artery-posterior inferior cerebellar artery end-to-end anastomosis: technical case report. Neurosurgery. 2006;59(suppl1):166-77.

7. Bertalanffy H, Gilsbach JM, Mayfrank L, Kawase T, Shiobara R, Toya S. Planning and surgical strategies for early management of vertebral artery and vertebrobasilar junction aneurysms. Acta Neurochir (Wien). 1995;134:60-5.

8. Choi $\mathrm{CH}, \mathrm{Cho} \mathrm{WH}$, Choi BK, Lee SW. Rerupture following endovascular treatment for dissecting aneurysm of distal anterior inferior cerebellar artery with parent artery preservation: retreatment by parent artery occlusion with Guglielmi detachable coils. Acta Neurochir (Wien). 2006;148:363-6.

9. Coert BA, Chang SD, Do HM, Marks MP, Steinberg GK. Surgical and endovascular management of symptomatic posterior circulation fusiform aneurysms. J Neurosurg. 2007;106: 855-65.

10. Cross DT, Moran CJ, Derdeyn CP, Mazumdar A, Rivet D, Chicoine MM. Neuroform stent deployment for treatment of a basilar tip aneurysm via a posterior communicating artery route. AJNR. 2005:26:2578-81.

11. Day DJ, Fukushima T, Giannotta SL. Cranial base approaches to posterior circulation aneurysms. J Neurosurg. 1997;87:544-54.

12. Debrun GM, Aletich VA, Thornton J, Allazzaz A, Charbel FT, Ausman JI, Bashir Q. Techniques of coiling cerebral aneurysms. Surg Neurol. 2000;53:150-6.

13. De Oliveira E, Rhoton Jr. AL, Peace D. Microsurgical anatomy of the region of the foramen magnum. Surg Neurol. 1995;24:293-352.

14. Drake CG. Bleeding aneurysms of the basilar artery. Direct surgical management in four cases. 1961. Can J Neurol Sci. 1999;26:35-40.

15. Drake CG, Peerless SJ. Giant fusiform intracranial aneurysms: review of 120 patients treated surgically from 1965 to 1992. J Neurosurg. 1997;87:141-62.

16. Figueiredo EG, Zabramski JM, Deshmukh P, Crawford NR, Preul MC, Spetzler RF. Anatomical and quantitative descrip- tion of the transcavernous approach to interpeduncular and prepontine cisterns. Technical note. J Neurosurg. 2006;104:957-64.

17. Fox AJ, Drake CG. Detachable balloon embolization for intracranial aneurysms. J Neurosurg. 1990;73:157-9.

18. Fukuyama T. The Sugita clip. Innovations in Neurosurgery. Japan Medical Culture Research Institute, Kawanishi Holdings, Inc. Japan, Ima Okayama; 2006. p.1-230.

19. George B, Dematons C, Cophignon J. Lateral approach to the anterior portion of the foramen magnum. Surg Neurol. 1988;29:484-90.

20. Gilsbach JM, Eggert HR, Seeeger W. The dorsolateral approach in ventrolateral craniospinal lesions. In: Voth $D$, Glees P, editors. Diseases in the cranio-cervical junction. Berlin: Walter de Gruyter; 1987. p.359-64.

21. Gonzalez LF, Amin-Hanjani S, Bambakidis NC, Spetzler RF. Skull base approaches to the basilar artery. Neurosurg Focus. 2005;19:E3.

22. Hakuba A, Liu S, Nishimura S. The orbitozygomatic infratemporal approach: a new surgical technique. Surg Neurol. 1986;26:271-6.

23. Hakuba A. Transcondylar approach. In: Hakuba A, Ohata K, Baba M, editors. Surgical anatomy of the skull base. Tokyo: Miwa Shoten; 1996. p.141-73.

24. Hashi K, Hakuba A, Ikuno H, Nishimura S. A midline vertebral artery aneurysm operated via transoral transclival approach. No Shinkei Geka. 1976;4:183-9.

25. Hernesniemi J, Ishii K, Karatas A, Kivipelto L, Niemel AM, Nagy L, Shen $\mathrm{H}$. Surgical technique to retract the tentorial edge during subtemporal approach: technical note. Neurosurgery. 2005;57:E408

26. Heros R. Lateral suboccipital approach for vertebral and vertebrobasilar artery lesions. J Neurosurg. 1986;64:559-62.

27. Inamasu J, Suga S, Sato S, Onozuka S, Kawase T. Longterm outcome of 17 cases of large-giant posterior fossa aneurysm. Clin Neurol Neurosurg. 2000;102:65-71.

28. Irie K, Murayama Y. Current endovascular therapy of cerebral aneurysm. No Shinkei Gueka. 2005;57:727-36.

29. James Ling A, D'Urso PS, Madan A. Simultaneous microsurgical and endovascular management of multiple cerebral aneurysms in acute subarachnoid haemorrhage. J Clin Neurosci. 2006;13:784-8.

30. Kato $\mathrm{Y}$, Sano H, Zhou J, Yamaguchi S, Kawase T, Yokoyama T, Kanno T. Deep hypothermia cardiopulmonary bypass and direct surgery of two large aneurysms at the vertebro-basilar junction. Acta Neurochir (Wien). 1996;138:1057-66.

31. Kawase T. Light up the "no-man's land" on the brain stem. Keio J Med. 1995; 44:133-9.

32. Kawase T, Toya S, Shiobara R, Mine T. Transpetrosal approach for aneurysms of the lower basilar artery. J Neurosurg. 1985;63:857-61.

33. Khandelwal P, Kato Y, Sano H, Yoneda M, Kanno T. Treatment of ruptured intracranial aneurysms: our approach. Minim Invasive Neurosurg. 2005;48:325-9.

34. Krisht AF, Krayenbuhl N, Sercl D, Bikmaz K, Kadri PA. Results of microsurgical clipping of 50 high complexity basilar apex aneurysms. Neurosurgery. 2007;60:242-50.

35. Lü J, Zhu X. Microsurgical anatomy of interpeduncular cistern and related arachnoid membranes. J Neurosurg. 2005;103:337-41.

36. Lü J, Zhu X. Characteristics of distribution and configuration of intracranial arachnoid membranes. Surg Radiol Anat. 2005;27:472-81. 
37. Mac Donald JD, Antonelli P, Day AL. The anterior subtemporal, medial transpetrosal approach to the upper basilar artery and ponto-mesencephalic junction. Neurosurgery. 1998;43:84-9.

38. Massad MG, Charbel FT, Chaer R, Geha AS, Ausman JI. Closed chest hypothermic circulatory arrest for complex intracranial aneurysms. Ann Thorac Surg. 2001;71:1900-4.

39. Mercado R, Santos-Franco J, Ortiz-Velazquez I, Gómez-Llata S. Vascular anatomy of the foramen of Vicq d'Azyr: a microsurgical perspective. Minim Invasive Neurosurg. 2004;47:102-6.

40. Meyer FB. Lateral suboccipital approach. In: Meyer FB, editor. Atlas of neurosurgery. Basic Approaches to cranial and vascular procedures. Edinburgh: Churchill Livingstone; 1999. p.255-7

41. Nakase $H$, Shin $Y$, Kanemoto $Y$, Ohnishi $H$, Morimoto $T$, Sakaki T. Long-term outcome of unruptured giant cerebral aneurysms. Neurol Med Chir (Tokyo). 2006;46:379-86.

42. Pai BS, Varma RG, Kulkarni RN, Nirmala S, Manjunath LC, Rakshith S. Microsurgical anatomy of the posterior circulation. Neurol India. 2007;55:31-41.

43. Paladino J, Mrak G, Mikliiäv P, Jednacak H, Mihaljeviäv D. The keyhole concept in aneurysm surgery - a comparative study: keyhole versus standard craniotomy. Minim Invasive Neurosurg. 2005;48:251-8.

44. Peerless SJ, Hernesniemi JA, Gutman FB, Drake CG. Early surgery for ruptured vertebrobasilar aneurysms. J Neurosurg. 1994;80:643-9.

45. Perneczky A. The posterolateral approach to the foramen magnum. In: Samii M, editor. Surgery in and around the brain stem and the third ventricle. Berlin: Springer; 1986. p.460-6.

46. Pitelli SD, Almeida GG, Nakagawa EJ, Marchese AJ, Cabral ND. Basilar aneurysm surgery: the subtemporal approach with section of the zygomatic arch. Neurosurgery. 1986;18:125-8.

47. Quiñones-Hinojosa A, Alam M, Lyon R, Yingling CD, Lawton MT. Transcranial motor evoked potentials during basilar artery aneurysm surgery: technique application for 30 consecutive patients. Neurosurgery. 2004;54:916-24

48. Rhim JR, Sheen SH, Oh SH, Noh JS, Chung BS. Aneurysm of the posterior inferior cerebellar artery: clinical features and surgical results. J Korean Neurosurg Soc. 2005;37:399-404.

49. Rice BJ, Peerless SJ, Drake CG. Surgical treatment of unruptured aneurysms of the posterior circulation. J Neurosurg. 1990;73:165-7.

50. Saito I, Takahashi H, Joshita H, Usui M, Sasaki T, Sano K. Clipping of vertebro-basilar aneurysms by the transoral transclival approach. Neurol Med Chir (Tokyo). 1980;20: 753-8.

51. Sano K. Temporo-polar approach to aneurysms of the basilar artery at and around the distal bifurcation: technical note. Neurol Res. 1980;2:361-7.

52. Sano K, Jinbo M, Saito I. Vertebrobasilar aneurysms, with special reference to the transpharyngeal approach to basilar artery. No Shinkei Geka. 1966;18:1197-203.

53. Sano K, Shiokawa Y. The temporo-polar approach to basilar artery aneurysms with or without zygomatic arch translocation. Acta Neurochir (Wien). 1994;130:14-9.

54. Sato $S$, Sato M, Oizumi T, Nishizawa M, Ishikawa M, Inamasu G, Kawase T. Removal of anterior clinoid process for basilar tip aneurysm: clinical and cadaveric analysis. Neurol Res. 2001;23:298-303.

55. Segawa H, Kohno M, Nakatomi H, Sano K, Saito I, Shiokawa Y. New aneurysm clip and applier for narrow spaces: technical note. Neurosurgery. 1999;45:939-42.

56. Sekhar LN, Natarajan SK, Britz GW, Ghodke B. Cerebral bypass and vascular reconstructions for posterior circulation aneurysms. In: Ramina R, Pires de Aguiar PH, Tatagiba M, editors. Samii’s essentials in neurosurgery. Berlin: Springer Verlag; 2008. p.353-74.

57. Shimokawara T, Shimomura T, Okumura Y, Sakaki T. Three cases of de novo aneurysms No Shinkei Geka. 2007;35: 365-70.

58. Spetzler R, Riina HA, Lemole Jr. GM. Giant Aneurysms. Neurosurgery. 2001;49:902-8.

59. Steinberg GK, Drake CG, Peerless SJ. Deliberate basilar or vertebral artery occlusion in the treatment of intracranial aneurysms. Immediate results and long-term outcome in 201 patients. J Neurosurg. 1993;79:161-73.

60. Sugita K. Aneurysms of the posterior circle of Willis. In: Sugita K, editor. Microneurosurgical atlas. Berlin: SpringerVerlag; 1985. p.62-135.

61. Suzuki J, Hori S, Iwabuchi T, Tanaka T. Surgical experience with aneurysm of the basilar artery. No Shinkei Gueka. 1996;18:1191-5.

62. Suzuki J, Iwabuchi T, Takaku A, Hori S, Harada N. Direct surgery of brain aneurysm-experiences with 103 cases. No Shinkei Gueka. 1967;19:815-24.

63. Suzuki J, Hori S, Sakurai Y. Intracranial aneurysms in the neurosurgical clinics in Japan. J Neurosurg. 1971;35:34-9

64. Suzuki J, Fujiwara S. Surgical treatment of intracranial giant aneurysms. No Shinkei Geka. 1988;16:341-50.

65. Tanaka Y, Kobayashi S, Hongo K, Tada T, Nagashima H, Kakizawa Y. Intentional body clipping of wide-necked basilar artery bifurcation aneurysms. J Neurosurg. 2000;93:169-74.

66. Tedeschi H, Rhoton Jr. AL. Lateral approaches to the petroclival region. Surg Neurol. 1994;41:180-216.

67. Thornton JA, Bashir Q, Alevitch VA, Debrum GM, Ausman JI, Charbel FT. What percentage of surgically clipped intracranial aneurysms have residual necks. Neurosurgery. 2000;46: 1294-300.

68. Torche M, Mahmood A, Araujo R, Dujovny M, Dragovic L, Ausman JI. Microsurgical anatomy of the lower basilar artery. Neurol Res. 1992;14:259-62.

69. Wen HT, Rhoton AL, Katsuta T, De Oliveira E. Microsurgical anatomy of the transcondylar, supracondylar, and paracondylar extensions of the far-lateral approach. J Neurosurg. 1997;87:555-85.

70. Yamamoto S, Hakuba A. Differences between transcondylar approach and retroauricular transpetrosal transtentorial approach for midline vertebrobasilar aneurysms. No Shinkei Geka. 1994;22:705-13.

71. Yonekawa Y. Operative neurosurgery: personal view and historical backgrounds. Anterior circulation — pterional approach. No Shinkei Geka. 2007;35:703-18

72. Youssef AS, Abdel Aziz KM, Kim EY, Keller JT, Zuccarello M, Van Loveren HR. The carotid-oculomotor window in exposure of upper basilar artery aneurysms: a cadaveric morphometric study. Neurosurgery. 2004;54:1181-9.

73. Yuki I, Murayama Y, Viñuela F. Endovascular management of dissecting vertebrobasilar artery aneurysms in patients presenting with acute subarachnoid hemorrhage. J Neurosurg. 2005;103:649-55

Original recebido em janeiro de 2009

Aceito para publicação em junho de 2009

Endereço para correspondência

Adriana Tahara

Rua Albuquerque Lins, 1.328

01230-000 - São Paulo, SP

E-mail: adrianatahara@yahoo.com.br 